International Mathematical Forum, 1, 2006, no. 37, 1819-1824

\title{
Characters and quasi-permutation representations of finite $p$-groups with few non-normal subgroups
}

\author{
Houshang Behravesh and Sebar Ghadery \\ Department of Mathematics \\ University of Urmia \\ Urmia, Iran \\ h.behravesh@mail.urmia.ac.ir
}

\begin{abstract}
Let $G$ be a finite group and let $\nu(G)$ denote the number of conjugacy classes of non-normal subgroups of $G$. In [1], we gave algorithms to calculate $c(G), q(G)$ and $p(G)$ for a finite group $G$. In this paper, we will calculate irreducible characters of finite $p$-groups $G$, with $\nu(G)=3$. Also we will calculate $c(G), q(G)$ and $p(G)$. Finally we will show that $\operatorname{cd}(G)=\{1, p\}$, where $\operatorname{cd}(G)$ denote the degree of irreducible characters of $G$.
\end{abstract}

Keywords : Quasi-permutation representations, Finite groups, Finite $p$ groups, Character theory.

Mathematics Subject Classification: Primary 20C15, Secondary 20B05

\section{INTRODUCTION}

Let $G$ be a finite group. Following [3] and [7], we let $\nu(G)$ denote the number of conjugacy classes of non-normal subgroups of $G$. Clearly $\nu(G)=0$ if and only if $G$ is a hamiltonian. In [3], R. Brandl characterizes the finite groups $G$ whose non-normal subgroups are all conjugate. In [7], H. Mousavi characterizes the finite groups $G$ with $\nu(G)=2$. In [8], H. Mousavi characterizes the finite p-groups $G$ with $\nu(G)=3$, and proves the following theorem: 
Theorem 1.1. Let $G$ be a finite p-group. Then $\nu(G)=3$ if and only if $G$ is isomorphic to one of the following groups:

(a) $G_{1}=Q_{8} \rtimes \mathbb{Z}_{4}$;

(b) $G_{2}=\left\langle x, y: x^{2}=y^{4}=\left[x, y^{2}\right]=1,[x, y]^{2}=y^{2}\right\rangle$;

(c) $G_{3}=\left\langle x, y: x^{8}=y^{8}=1, x^{4}=y^{4}, y x y=x\right\rangle$;

(d) $G_{4}=\left\langle x, y: x^{4}=y^{8}=1,[y, x]=y^{4} x^{2}\right\rangle$;

(e) $G_{5}=\left\langle x, y, z: x^{2}=y^{2}=z^{2^{n}}=[x, z]=[y, z]=1,[x, y]=z^{2^{n-1}}\right\rangle$, $n \geq 2$;

(f) $G_{6}=\left\langle x, y: x^{3^{2}}=y^{3^{n}}=1,[x, y]=y^{3^{n-1}}\right\rangle, n \geq 2$.

Now let define the quantities $c(G), q(G)$ and $p(G)$. By a quasi-permutation matrix we mean a square matrix over the complex field $\mathbb{C}$ with non-negative integral trace. Thus every permutation matrix over $\mathbb{C}$ is a quasi-permutation matrix. For a given finite group $G$, let $p(G)$ denote the minimal degree of a faithful permutation representation of $G$ (or of a faithful representation of $G$ by permutation matrices), let $q(G)$ denote the minimal degree of a faithful representation of $G$ by quasi-permutation matrices over the rational field $\mathbb{Q}$, and let $c(G)$ denote the minimal degree of a faithful representation of $G$ by complex quasi-permutation matrices. See [1]. It is easy to see that

$$
c(G) \leq q(G) \leq p(G)
$$

where $G$ is a finite group.

In this paper, we will calculate irreducible characters of finite $p$-groups $G$, with $\nu(G)=3$. Also we will calculate $c(G), q(G)$ and $p(G)$. Finally we will show that $\operatorname{cd}(G)=\{1, p\}$, where $\operatorname{cd}(G)$ denote the degree of irreducible characters of $G$.

\section{Quasi permutation Representation of groups $G_{1}, G_{2}, G_{3}, G_{4}$}

We will number our groups as they appear in the library of small groups in GAP.

In order to calculate $q(G)$ we will need the Schur index of irreducible characters. Hence we will need the following lemmas.

Lemma 2.1. Let $G$ be a 2-group and $\chi \in \operatorname{Irr}(G)$. Then $m_{\mathbb{Q}}(\chi)=m_{\mathbb{R}}(\chi)$.

Proof : See [[9], Satz 1]. 
Lemma 2.2. Let $G$ be a finite group and $\chi \in \operatorname{Irr}(G)$. Let

$$
\nu(\chi)=\frac{1}{|G|} \sum_{g \in G} \chi\left(g^{2}\right) .
$$

Then

$$
\nu(\chi)= \begin{cases}1 & \text { if } \chi=\bar{\chi} \text { and } m_{\mathbb{R}}(\chi)=1 \\ -1 & \text { if } \chi=\bar{\chi} \text { and } m_{\mathbb{R}}(\chi)=2 \\ 0 \text { if } \chi \neq \bar{\chi} & \end{cases}
$$

Proof : See [[4], page 191, Lemma 33.4].

Note : By Lemmas 2.1 and 2.2 one can calculate the Schur index of any irreducible character of a 2-group by calculating $\nu(\chi)$. Note that calculating $\nu(\chi)$ it is not so easy.

Lemma 2.3. Let $G$ be a 2-group with an irreducible character of degree 2. Then det $\chi$ is the principle character if and only if the Schur index $m_{\mathbb{Q}}(\chi)=2$.

Proof : See [[5], Theorem 3].

Theorem 2.4. The following table hold.

\begin{tabular}{|c|c|c|c|}
\hline$G$ & $c(G)$ & $q(G)$ & $p(G)$ \\
\hline$G_{1} \cong(32,26)$ & 8 & 12 & 12 \\
\hline$G_{2} \cong(16,8)$ & 8 & 8 & 8 \\
\hline$G_{3} \cong(32,15)$ & 16 & 16 & 16 \\
\hline$G_{4} \cong(32,12)$ & 12 & 12 & 12 \\
\hline
\end{tabular}

Proof : We will use the GAP for the character tables and the subgroups and the core of subgroups. Also we will use Lemmas 2.2 and 2.3 and $\nu(\chi)$ for Schur indices. Finally we will use [[1], Corollaries 2.4 and 3.11] for groups of cyclic center or [[1], Theorems 2.2 and 3.6] for groups of non-cyclic center, in order to calculate $c(G), q(G)$ and $p(G)$.

\section{Characters and Quasi-Permutation Representations of $G_{5}$}

Let

$$
G=G_{5}=\left\langle x, y, z: x^{2}=y^{2}=z^{2^{n}}=[x, z]=[y, z]=1,[x, y]=z^{2^{n-1}}\right\rangle .
$$


Then it is easy to see that $Z(G)=\langle z\rangle$ and $G^{\prime}=\left\langle z^{2^{n-1}}\right\rangle$. Also $|G|=2^{n+2}$ and $|G: Z(G)|=4$. Since $G$ is non-abelian, so by [[6], Corollary 2.30], $\operatorname{cd}(G)=$ $\{1,2\}$. By [[6], Corollary 2.6], we have $\left|G: G^{\prime}\right|=2^{n+1}$ linear characters. Also by [[6], Corollary 2.7] we have $2^{n-1}$ irreducible characters of degree 2 .

Theorem 3.1. (1) $G / G^{\prime} \cong C_{2} \times C_{2} \times C_{2^{n-1}}$.

(2) All characters of degree 2 are faithful. Also if $\chi_{i}$ denote an irreducible non-linear characters of $G$, then we have:

$$
\begin{aligned}
& \chi_{i}(x)=0 \text { for all } x \in G-Z(G) \\
& \chi_{i}\left(z^{j}\right)=2 \epsilon^{i j},
\end{aligned}
$$

where $1 \leq j \leq 2^{n},\left(2^{n}, i\right)=1,1 \leq i \leq 2^{n}-1$ and $\epsilon$ is an $2^{n}$-th primitive root of $2^{n}$.

(3) $c(G)=q(G)=p(G)=2^{n+1}$

Proof : (1) It is easy to see that $G / G^{\prime} \cong\left\langle x G^{\prime}, y G^{\prime}, z G^{\prime}\right\rangle$. So the result follows.

(2) By [[6], Theorem 2.32 (b)], there exists a faithful irreducible character of $G$. By (1), $G$ has no linear and faithful character. So the faithful character has degree 2. Also by [[6], Corollary 2.30], we know that this character vanishes on $G-Z(G)$ and on any element of $Z(G)$ is equal to 2 times the value of an irreducible character of $Z(G)$ on that element. As $Z(G)$ has only $2^{n-1}$ faithful linear characters and they are all in one Galios orbit, so $G$ has at least $2^{n-1}$ faithful and irreducible character of degree 2. Now by the first paragraph of this section the result follows.

(3) $\langle x\rangle$ is a subgroup of $G$ and its corefree. So by [[1], Corollary 2.4] and [[1], Theorem 4.12], the result follows.

\section{Quasi-Permutation Representations of $G_{6}$}

It is easy to prove that

$$
G_{6}=\left\langle x^{2}, y:\left(x^{2}\right)^{9}=y^{3^{n}}=1, x^{-2} y x^{2}=y^{1+3^{n}}\right\rangle .
$$

So

$$
G_{6} \cong\left\langle z, y: z^{9}=y^{3^{n}}=1, z^{-1} y z=y^{1+3^{n}}\right\rangle .
$$


Now it is easy to see that

$$
\left\langle z, y: z^{3^{2}}=y^{3^{n}}=1, z^{-1} y z=y^{1+3^{n}}\right\rangle
$$

satisfy the conditions of metacyclic $p$-groups stated in [[2], page 347]. So we are able to calculate the irreducible characters of $G_{6}$ and also show that

$$
c\left(G_{6}\right)=q\left(G_{6}\right)=p\left(G_{6}\right)=3^{2}+3^{n} .
$$

Also here we will state some results without using of [2].

Lemma 4.1. (Ito) Let $G$ be a finite group and let $H$ be an abelian subgroup of $G$. Then for all $\chi \in \operatorname{Irr}(G)$

$$
\chi(1)|| G: H \mid \text {. }
$$

Moreover if $G$ be a finite non-abelian p-group and $|G: H|=p$, then $\operatorname{cd}(G)=$ $\{1, p\}$.

Proof : See [[6], Theorem 6.15].

Lemma 4.2. Let $G=G_{6}=\left\langle x, y: x^{9}=y^{3^{n}}=1,[x, y]=y^{3^{n-1}}\right\rangle$. Then

(1) $Z(G)=\left\langle x^{3}, y^{3}\right\rangle,|Z(G)|=3^{n}$ and $|G: Z(G)|=9$.

(2) $G^{\prime}=\left\langle y^{3^{n-1}}\right\rangle$.

(3) $\operatorname{cd}(G)=\{1,3\}$.

Proof : (1) and (2) are easy to prove.

(3) Let $H=\left\langle x^{3}, y\right\rangle$. Then $H$ is an abelian group and $H \triangleleft G$. Since $\chi(1)|| G \mid$ for all irreducible characters of $G$, so $\chi(1)$ is a power of $p$ for non-linear and irreducible characters of $G$. Now by Lemma 4.1, degree of non-linear and irreducible characters of $G$ are 3 .

Also one can use the fact that $\chi(1) \leq|G: Z(G)|^{1 / 2}=3$ for all irreducible characters of $G$, so the result (3) follows.

Corollary 4.3. Let $G$ be a finite p-group and $\nu(G)=3$. Then $\operatorname{cd}(G)=\{1, p\}$.

\section{REFERENCES}

[1] H. Behravesh. Quasi-permutation representations of p-groups of class 2. J. London Math. Soc. (2) 55 (1997) 251-260.

[2] H. Behravesh. Quasi-permutation representations of metacyclic p-groups with noncyclic center. Southeast Asian Bulletin of Mathematics (2000) 24: 345-353. 
[3] R. Brandel. Groups with few non-normal subgroups. Comm. Algebra 23 (1995) 20912098.

[4] L. Dornhoff. Group representation theory, part A. Dekker, New York,1971.

[5] Y. Iiida. A note on the Schur index of an irreducible character of a 2-group. Soochow J. Math. Vol. 24, No. 2 (1998) 163-165.

[6] I. M. Isaacs. Character theory of finite groups. Dover Publications, Inc., New York, (1994).

[7] H. Mousavi. On finite groups with few non-normal subgroups. Comm. Algebra 27(7) (1999), 3143-31151.

[8] H. Mousavi. Finite p-groups with non-normal subgroups having the same order. Will appear.

[9] P. Roquette. Realisierung von Darstellungen endlicher nilpotenter Gruppen. Archiv. der Math. 9 (1958) 241-250.

[10] M. Schonert et al. GAP: Groups, Algorithms, and Programming. Lehrstuhl D für Mathematik, RWTH Aachen, 1994.

Received: February 1, 2006 